Red oil-droplets perform, incidentally, most of the functions of yellow filters--even better than the latter when the objective light intensity is very high, but introduce an undesirable curtailment of the range of the subjective chromatic spectrum, and become inefficient in "comfortable" illuminations except where the amount of scattered medium-wave light is great, as at the twilight periods. For these reasons they are never found alone, but always accompanied by large numbers of paler droplets which bear the brunt of the work of vision under ordinary conditions. The primary importance of red droplets, in birds, is in sharpening vision in the reddened illumination (with its accompaniment of scattered violet, blue, and green light) of their favourite matutinal period of great activity. In turtles (and the kingfisher?) their primary function is to reduce the excessive glare which interferes with sharp vision over water. A red oil-droplet is of no value in natural mist or under natural waters.

Those diurnal vertebrates which rely upon lenticular, corneal, macular, and capillary filters must of necessity choose a single optimum colour and density, and accept a resultant limitation of those activities in which their visual capacities are important. The oil-droplet type of filter, however, permits of the development of a functionally multiplex photopic apparatus, with a resultant wider range of adaptation to varying illumination conditions. Because many cones would not be maximally efficient under any one static set of conditions, only very cone-rich retinae can afford to evolve a multiplex oil-droplet mosaic-and it is in such retinae that the multiplex system finds its highest expression.

To be continued.

\title{
THE CILIARY MUSCLE AND DESCEMET'S MEMBRANE
}

\author{
BY
}

\section{H. DE Villiers}

LATE DISTRICT SURGEON, LAINGSBURG, SOUTH AFRICA

Sir Arthur Thomson in The Ophthalmoscope Vol. IX, 1911, described the action of the ciliary muscle on the scleral spur, at the angle of the anterior chamber, with a pump-like effect. It is probably a tribute to his work that to-day it is generally taught that the anterior attachment of the ciliary muscle is to the scleral spur.

(Vide: Cunningham's "Anatomy"; Gray's "Anatomy"; Buchanan-"Manual of Anatomy"; Duke-Elder-"Text-book of Ophthalmology I.") 
Now a study of the histology of the angle of the anterior chamber does not satisfy one that the anterior attachment of the ciliary muscle is to the posterior border of the scleral spur.

The ciliary muscle anteriorly ends in connective tissue. Much of this tissue is attached to the posterior border of the scleral spur, but a good deal passes straight forwards, avoiding the scleral spur altogether, towards the "end of Descemet's membrane."

From the anterior border of the scleral spur trabeculae pass forward to the "end of Descemet's membrane" and to the inner corneal lamellae.

Most authors admit freely that there are two separate sets of trabeculae at the angle of the anterior chamber medial to Schlemm's Canal, viz:-

(1) Scleral trabeculae running to the scleral spur, and

(2) Uveal trabeculae passing directly to the ciliary muscle.

(Henderson, Parsons, Salzmann, Fuchs, Rauber-Kopsch, Lyle and de Souza).

As regards the posterior attachment of the ciliary muscle, there is almost universal agreement that the posterior attachment is to the sclera of the posterior segment of the eye, although the choroid is pulled upon and moved forwards and backwards to some extent when the ciliary muscle contracts and relaxes.

Were the posterior attachment of the ciliary muscle merely to the choroid this vascular organ would be torn by the pull exerted on it.

The ciliary muscle exhibits a diversity of functions that must be well-nigh unique. Its work as regards the accommodation of the eye is sufficiently appreciated. It is probably worth while, however, to refer to the striking analogy between its posterior and anterior attachments.

Posteriorly, the ciliary muscle

(1) is attached directly to the whole of the posterior portion of the eye.

(2) A neighbouring tissue is interposed between the muscle and its ultimate attachment, viz., the choroid.

(3) The choroid has sufficient connection with the fibres of the posterior attachment to cause its anterior portion to move with the fibres, and to dilate and to collapse as the ciliary muscle contracts and relaxes-a matter of the greatest importance to the vascular circulation of the eye.

Anteriorly, the ciliary muscle

(1) is attached directly, according to our view, to the whole of the cornea. 
(2) A neighbouring tissue is interposed between the muscle and its ultimate attachment, viz., the scleral spur.

(3) The scleral spur has sufficient connection with the fibres of the anterior attachment to cause its anterior portion to move with

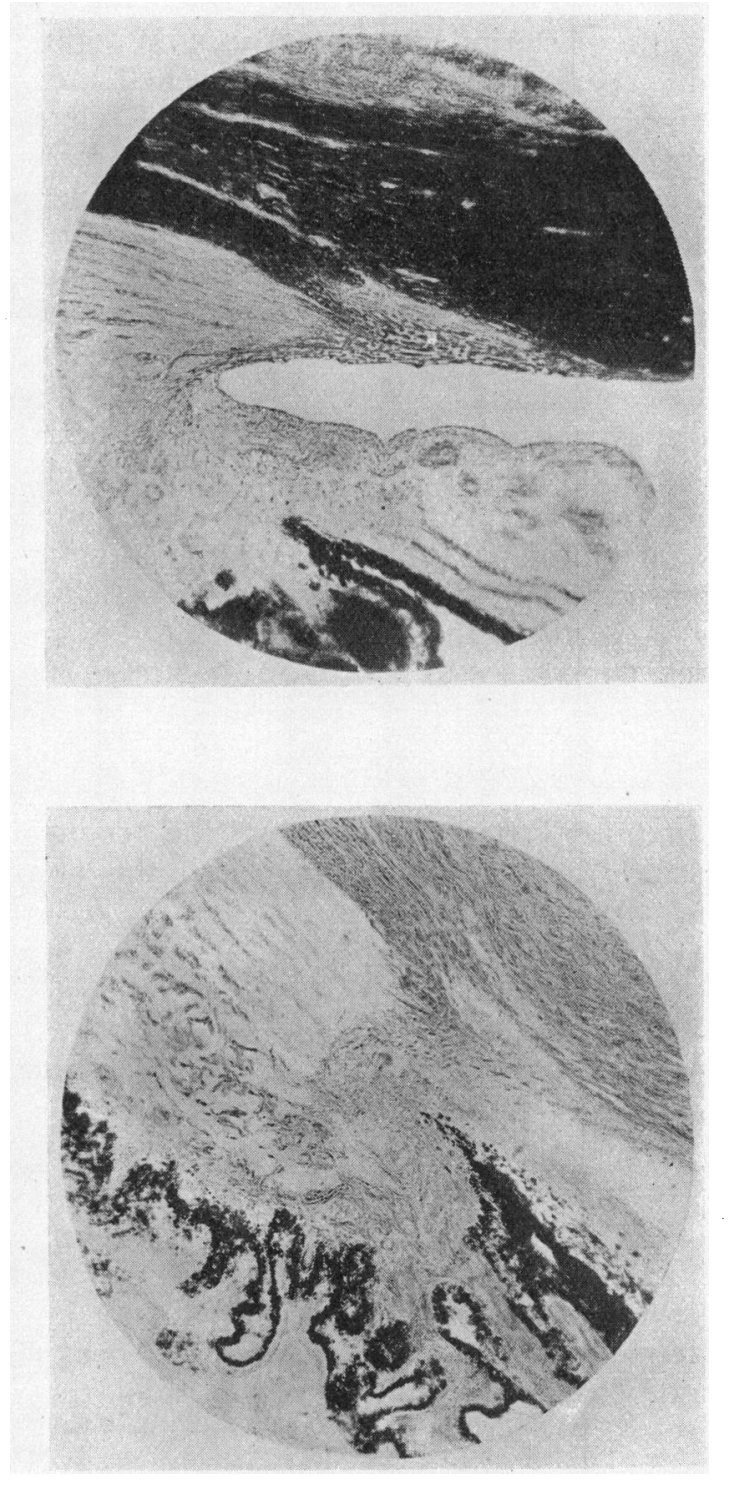

Two photographs showing the structures at the angle of the Anterior Chamber. 
the fibres as the ciliary muscle contracts and relaxes, thereby causing the canal of Schlemm to dilate and to collapse-a matter of the greatest importance to the intra-ocular fluid circulation of the eye, especially in cases of raised intra-ocular tension.

We would suggest then that the anterior attachment of the ciliary muscle should not be regarded as being to the scleral spurany more than the posterior attachment is now regarded as being to the choroid. Were the ciliary muscle attached anteriorly merely to the scleral spur, without the support of the scleral trabeculae, we would expect that the scleral spur would probably be torn, just as in the case of the choroid, when the muscle contracts.

In Collins and Mayou "Pathology and Bacteriology of the Eye," p. 347, a similar condition is actually described where the ciliary muscle loses its most fixed point of attachment and the ciliary body is displaced backwards when the ligamentum pectinatum is ruptured.

More accurately the whole of the cornea should be regarded as being the anterior attachment of the ciliary muscle.

Descemet's Membrane is described by Salzmann as being a typical glass membrane-a highly refractile structureless homogeneous membrane of great firmness and elasticity. Descemet's membrane is $5-7 \mu$ thick in the centre part. Towards the angle of the chamber it increases to $8-10 \mu$ in thickness.

Salzmann further describes the thickened end of Descemet's membrane as breaking up and splaying out to help form the trabeculae.

Gray's "Anatomy" states definitely "the triangular mass of trabecular tissue ... . is continuous with the posterior elastic lamina of the cornea."

Parsons agrees with this view in his "Diseases of the Eye."

Fuchs in his text book (15th Edition) regards Descemet's membrane as being a part of the uveal tract.

Collins and Mayou (p. 78) state that "the fibres of the ligamentum pectinatum stain and react in precisely the same way as the hyaline layer of Descemet's membrane and, like it, are probably the product of the cells which ultimately line them."

In view of the evidence quoted above together with the description of the histology of the parts under discussion the question arises whether Descemet's membrane should not be regarded as an anterior tendon of the ciliary muscle. That it could be a "tendon" modified for the optical purposes of the eye, just as the opaque sclera is modified to become the clear transparent cornea, is not incompatible with its nature, structure, position, proportions, connections, histological staining reactions, mesodermal origin, and embryological development. 
In considering this question it should be remembered that the eye is capable of rude departures from recognised histological rules-we mention two of these-the dilator muscle arising from ectodermal tissue, and the growth throughout life of the lens, an ectodermal tissue, without ever casting off any of its cells.

I am indebted to Mr. H. B. Stallard for his kindness in allowing me to make free use of the pathological laboratory at Moorfields.

\title{
THE DIAGNOSIS OF IRIDO-CILIARY TUBERCULOSIS
}

\author{
BY \\ HENRI LAGRANGE* \\ CHIEF OPHTHALMOLOGIST, PARIS HOSPITAL SERVICES
}

BEFORE entering upon discussion of the report on the direct and differential diagnosis of tuberculosis of the iris and ciliary body, I desire to present this problem in a few words as illuminated by the papers which I have had the honour of classifying and abstracting for your information.

\section{I.-Ocular Tuberculosis is a Secondary Lesion supposedly due to Superinfection}

Publication of the first case of iridian tuberculosis by Gradenigo and Richetti, in 1867, was immediately followed by a discussion on the pathogenesis which has only recently been really closed. It was necessary to determine. whether irido-ciliary tuberculosis was a primary or a secondary effect.

Many collected cases seemed to indicate that this affection was primary. Denig, for example, collected, in 1885, 38 cases of localized ocular tuberculosis not having any evident previous history of tuberculosis. With these cases he compared systematic examination of 60 cases of pulmonary tuberculosis, 90 cases of bone tuberculosis, 20 cases of glandular tuberculosis and 20 cases of various types of tuberculosis. In all of these comparative cases, only five showed ocular metastasis.

In contrast with this finding, however, was the opinion stated by de Wecker, in France, Leber, in Germany, and Fuchs, in Austria,

* Introduction to discussion of my report on the direct and differential diagnosis of tuberculosis of the iris and ciliary body. International Congress on Ophthalmology, Madrid, April 16, 1933 . Cf. " Tuberculosis of the Iris and Ciliary Body," published by G. Doin \& Company, Paris, 1933. 